\title{
FAUNA FLEBOTOMÍNICA (DIPTERA, PSYCHODIDAE) ENCONTRADA NO MUNICÍPIO DE PIRENÓPOLIS, GOIÁS, BRASIL, NO PERÍODO DE 2005 A 2010
}

\author{
SANDFLIES (DIPTERA, PSYCHODIDAE) FOUND IN PIRENÓPOLIS, GOIÁS, \\ BRAZIL DURING THE 2005-2010 PERIOD
}

\author{
M. SANTALUCIA ${ }^{1}$, W. A. BEZERRA ${ }^{1}$, J. M. D’ABADIA ${ }^{1}$, C. D. DA SILVA ${ }^{1}$, N. G. COSTA ${ }^{1}$, \\ T. S. A. BASTOS ${ }^{2 *}$, D. M. C. MADRID ${ }^{2}$
}

\begin{abstract}
RESUMO
Contínuos casos humanos e caninos de LV e LTA detectados em Pirenópolis (Goiás), cidade turística com intenso fluxo de pessoas por suas atrações ecológicas, religiosas e históricas, chamaram a atenção para a investigação e reunião de dados neste local. Entre os anos de 2005 a 2010, foram instaladas armadilhas luminosas tipo CDC (em ambiente intra e peridomiciliar) para investigar a presença e identificar as espécies de flebotomíneos existentes nos locais onde casos de LV e LTA foram detectados. De um total de 551 armadilhas instaladas, 205 (37,2\%) não capturaram flebotomíneos. Nas demais, capturou-se 5.648 espécimes, distribuídos em 20 diferentes espécies: Lutzomyia longipalpis (53,3\%), L. whitmani $(43,3 \%)$, L. lenti $(1,3 \%)$, L. sordellii $(0,8 \%)$, L termitophila $(0,2 \%)$, L. salesi $(0,19 \%), L$. acanthopharynx $(0,15 \%)$, L. lutziana $(0,08 \%)$, L. pressatia $(0,08 \%)$, L. quinquefer $(0,08 \%)$, Brumptomyia sp $(0,07 \%)$, L. davisi $(0,07 \%)$, L. aragaoi $(0,07 \%)$, L. pessoai $(0,05 \%)$, B. avelari $(0,03 \%)$, L. oliveirai $(0,01 \%)$, L baculus $(0,01 \%)$, L. evandroi $(0,01 \%)$, L. shannoni $(0,01 \%)$ e $L$. carmelinoi $(0,01 \%)$. O encontro destes flebotomíneos, machos e fêmeas, indicam que há reprodução das espécies no local. A ocorrência dos vetores da LV (L. longipalpis) e de LTA ( $L$. whitmani) indicam risco de transmissão da doença a humanos e cães (reservatório da LV) no município. Desta forma, faz-se necessária uma especial atenção do serviço de vigilância epidemiológica no local para adotar as medidas de controle e prevenção adequadas, assim como a orientação da população e articulação com outros órgãos públicos e privados parar viabilizar ações educativas e de manejo ambiental.
\end{abstract}

PALAVRAS-CHAVE: GOIÁS. LEISHMANIA. LEISHMANIOSE.

\footnotetext{
${ }^{1}$ Laboratório de Saúde Pública Dr. Giovanni Cysneiros - LACEN-GO, 74.853-120 Goiânia, GO, Brasil

${ }^{2}$ Programa de Pós-graduação em Ciência Animal. Escola de Veterinária e Zootecnia/UFG.

*Tsabvet@gmail.com
} 